University of Wollongong

Research Online

Faculty of Engineering - Papers (Archive)

Faculty of Engineering and Information

Sciences

$1-1-2012$

\title{
A "skeleton/skin"strategy for preparing ultrathin free-standing single-walled carbon nanotube/polyaniline films for high performance supercapacitor electrodes
}

Zhiqiang Niu

Chinese Academy Of Sciences

Pingshan Luan

Chinese Academy Of Sciences, Beijing, China

Qi Shao

Nanyang Technological University, Singapore

Haibo Dong

Chinese Academy Of Sciences

Jinzhu Li

Chinese Academy Of Sciences

See next page for additional authors

Follow this and additional works at: https://ro.uow.edu.au/engpapers

Part of the Engineering Commons

https://ro.uow.edu.au/engpapers/5015

\section{Recommended Citation}

Niu, Zhiqiang; Luan, Pingshan; Shao, Qi; Dong, Haibo; Li, Jinzhu; Chen, Jun; Zhao, Duan; Cai, Le; Zhou, Weiya; Chen, Xiaodong; and Xie, Sishen: A "skeleton/skin"strategy for preparing ultrathin free-standing single-walled carbon nanotube/polyaniline films for high performance supercapacitor electrodes 2012, 8726-8733.

https://ro.uow.edu.au/engpapers/5015

Research Online is the open access institutional repository for the University of Wollongong. For further information contact the UOW Library: research-pubs@uow.edu.au 


\section{Authors}

Zhiqiang Niu, Pingshan Luan, Qi Shao, Haibo Dong, Jinzhu Li, Jun Chen, Duan Zhao, Le Cai, Weiya Zhou, Xiaodong Chen, and Sishen Xie 


\author{
Cite this: Energy Environ. Sci., 2012, 5, 8726
}

wWw.rsc.org/ees

PAPER

\title{
A "skeleton/skin" strategy for preparing ultrathin free-standing single-walled carbon nanotube/polyaniline films for high performance supercapacitor electrodes
}

\author{
Zhiqiang Niu, ${ }^{a b}$ Pingshan Luan, ${ }^{a d}$ Qi Shao, ${ }^{b}$ Haibo Dong, ${ }^{a d}$ Jinzhu Li,${ }^{a d}$ Jun Chen, ${ }^{c}$ Duan Zhao, ${ }^{a d}$ Le Cai, ${ }^{a d}$ \\ Weiya Zhou, ${ }^{* a}$ Xiaodong Chen ${ }^{* b}$ and Sishen $\mathrm{Xie}^{a}$
}

Received 25th April 2012, Accepted 30th July 2012

DOI: $10.1039 / \mathrm{c} 2 \mathrm{ee} 22042 \mathrm{c}$

One of the most critical aspects in the preparation of single-walled carbon nanotubes (SWCNTs)/ conducting polymer hybrid electrodes is to improve the energy density without seriously deteriorating their high power capability. Here, we report a "skeleton/skin" strategy for the preparation of freestanding, thin and flexible SWCNT/polyaniline (PANI) hybrid films by a simple in situ electrochemical polymerization method using directly grown SWCNT films with a continuous reticulate structure as template. In situ electrochemical polymerization can achieve effective deposition of PANI onto the surface of SWCNT bundles in the films and control the morphology and microstructure of the SWCNT/PANI hybrid films. In a SWCNT/PANI hybrid film, the directly grown SWCNT film with continuous reticulate architecture acts as the skeleton and PANI layers act as the skin. This unique continuous "skeleton/skin" structure ensures that these hybrid films have much higher conductivity compared to SWCNT/PANI composite films based on post-deposition SWCNT films. Flexible supercapacitors have been fabricated using the SWCNT/PANI hybrid films as both electrodes and charge collectors without metallic current collectors. High energy and power densities $\left(131 \mathrm{~W} \mathrm{~h} \mathrm{~kg}^{-1}\right.$ and $62.5 \mathrm{~kW} \mathrm{~kg}^{-1}$, respectively) have been achieved for the optimized assembly. The high electrical conductivity and flexibility, in combination with continuous porous architecture, suggests that the asprepared ultrathin free-standing SWCNT/PANI hybrid films have significant potential as promising electrode materials for thin, lightweight and flexible energy storage devices with high performance.

${ }^{a}$ Beijing National Laboratory for Condensed Matter Physics, Institute of Physics, Chinese Academy of Sciences, Beijing 100190, China. E-mail: wyzhou@iphy.ac.cn; Fax: +86-10-82640215; Tel: +86-10-82649381

${ }^{b}$ School of Materials Science and Engineering, Nanyang Technological University, 50 Nanyang Avenue, Singapore 639798. E-mail: chenxd@, ntu.edu.sg
'Intelligent Polymer Research Institute, ARC Centre of Excellence for Electromaterials Science, Australian Institute of Innovative Materials, Innovation Campus, University of Wollongong, Northfields Avenue, Wollongong, NSW 2522, Australia

${ }^{d}$ Graduate School of the Chinese Academy of Sciences, Beijing 100039, China

\section{Broader context}

The hybrid electrodes of SWCNT/conducting polymer display high energy density due to pseudocapacitance originating from the conducting polymer. However, their power density is dramatically reduced in comparison with pure SWCNT-based electrodes, due to the poor electrical conductivity of PANI layers and overlapped PANI-PANI contact. Therefore, one of the most critical aspects in the development of SWCNT/conducting polymer supercapacitors is to optimize the energy density without deteriorating their high power capability as these two parameters determine concomitantly the ultimate performance of the supercapacitor. In this work, we report a "skeleton/skin" strategy to prepare free-standing, thin and flexible SWCNT/PANI hybrid films by a simple in situ electrochemical polymerization method using directly grown SWCNT films with continuous reticulate structure as template. The high electrical conductivity and flexibility, in combination with continuous porous architecture, suggest that as-prepared ultrathin freestanding SWCNT/PANI hybrid films have significant potential as promising electrode materials for thin, lightweight and flexible energy storage devices with high performance. The flexible supercapacitors based on the SWCNT/PANI hybrid films achieve high energy and power densities. 


\section{Introduction}

Supercapacitors, also known as electrochemical capacitors, have attracted a lot of attention because of their high power and energy density as well as long cycle life. ${ }^{1-4}$ As energy storage devices, supercapacitors can be applied to many fields, such as electric vehicles, pulsed power applications and portable devices. $^{2}$ For such applications, it is extremely important to develop supercapacitors with both higher power density and higher energy density than those currently available. The performance characteristics of supercapacitor devices are fundamentally determined by the structures and electrochemical properties of electrode materials. Carbon-based materials are widely used as supercapacitor electrodes because of their desirable physical and chemical properties. ${ }^{3,5-7}$ Among these carbonbased materials, single-walled carbon nanotubes (SWCNTs) have attracted a great deal of attention owing to their high conductivity, low mass density, large specific surface area and high mechanical strength. ${ }^{8-22}$ Pure SWCNT film-based supercapacitor electrodes are usually regarded as a competitive material for high-power electrodes because of their good electrical conductivity and readily accessible surface area. ${ }^{11,14,15}$ However, the specific surface area of the pure SWCNT filmbased electrodes is generally low because SWCNTs exist in the form of bundles, leading to a low energy density. ${ }^{3,23}$ Much effort has gone into improving the energy density of SWCNT-based electrodes, such as adding conducting polymers into SWCNTbased electrodes. ${ }^{1724-34}$ The SWCNT/conducting polymer electrodes display high energy density due to pseudocapacitance originating from the conducting polymer, but, their power density is dramatically reduced in comparison to the pure SWCNT-based electrodes, due to the poor electrical conductivity of conducting polymers. Therefore, one of the most critical aspects in the development of SWCNT/conducting polymer supercapacitors is to optimize the energy density without deteriorating their high power capability, as these two parameters determine the ultimate performance of the supercapacitors.

Polyaniline (PANI) is considered to be one of the most promising electrode materials because of its relatively high conductivity and lower cost compared to many other conducting polymers. ${ }^{35-40}$ Recently, significant research efforts have focused on preparation of CNT/PANI composites, wherein the PANI is coated onto the surface of CNTs or bundles of CNTs in the form of powders or films by various approaches, such as electropolymerization and chemical oxidation polymerization..$^{29-32,41}$ Electrodes based on SWCNT/PANI powders have usually been achieved by pressing the composites into tablets or by mixing SWCNT/PANI composite with conductive binders and coating it onto collector electrodes. ${ }^{17,42,43}$ In these electrodes, the connections between SWCNT/PANI nanowires or nanorods overlap with the PANIPANI contact. Compared to SWCNT-SWCNT contact, the overlapped PANI-PANI contact has a higher sheet resistance, leading to a lower power density of such SWCNT/PANI composite electrodes. Besides, the brittle mechanical nature of SWCNT/PANI composite electrodes hinders their practical applications. For the electrodes of mixed SWCNT/PANI composites and binders, the addition of binders degraded the electrical and electrochemical properties of SWCNT/PANI electrodes. ${ }^{3}$ These SWCNT/PANI composite electrodes cannot meet the future demands of supercapacitors, which are required to be thin, lightweight, cheap and flexible. Several groups have investigated the fabrication of flexible CNT/PANI films by different methods with the assistance of CNT film templates. ${ }^{30,31,41}$ For instance, Fan et al. reported that the free-standing CNT/PANI film can be achieved by chemical oxidation polymerization using CNT Bucky paper and freestanding CNT networks with randomly entangled individual CNTs and CNT bundles as template. ${ }^{31,41}$ They assembled all-solid-state paperlike polymer ( $\sim 30 \mu \mathrm{m}$ in thickness for either electrode component) supercapacitors with high specific capacitance. ${ }^{41}$ Sun et al. demonstrated the preparation of free-standing CNT/PANI film by electropolymerization using CNT Bucky paper as template. ${ }^{30}$ However, the conductivity of these free-standing CNT/PANI films was generally lower than $150 \mathrm{~S} \mathrm{~cm}^{-1} .^{30,31} \mathrm{In}$ other words, although the energy densities of these free-standing CNT/PANI films was improved due to pseudocapacitance originating from PANI, the power densities of these free-standing CNT/PANI film electrodes was generally lower than $2.2 \mathrm{~kW} \mathrm{~kg}{ }^{-1}{ }^{31,41}$ Furthermore, the thickness of CNT/PANI film is still tens of micrometers. Therefore, fabricating large-area, thin, lightweight and flexible SWCNT/PANI composite film electrodes with high conductivity would satiate a large demand. Here, we report a "skeleton/skin" strategy to prepare thin and flexible SWCNT/PANI hybrid films with high conductivity, in which directly grown SWCNT film with continuous reticulate architecture acts as the skeleton and PANI acts as the skin. Thin, lightweight and flexible supercapacitors have been fabricated using as-prepared SWCNT/PANI hybrid films as both electrodes and charge collectors without metallic current collectors. Excellent performance in terms of high energy and power densities has been achieved.

\section{Experimental}

\section{Preparation of SWCNT/PANI hybrid films}

The SWCNT films used as template to electrodeposit PANI were directly prepared by a floating chemical vapor deposition method, as reported previously..$^{45}$ Electrodeposition of PANI was performed in a traditional three-electrode cell, in which a platinum plate, a saturated Calomel electrode (SCE) and a SWCNT film were used as the counter, reference and working electrodes, respectively, as shown in Fig. 1a. PANI was electrodeposited at a constant current of $1 \mathrm{~mA} \mathrm{~cm}{ }^{-2}$ versus $\mathrm{SCE}$ in an electrolyte of $0.5 \mathrm{M} \mathrm{H}_{2} \mathrm{SO}_{4}, 0.5 \mathrm{M}$ $\mathrm{Na}_{2} \mathrm{SO}_{4}$ and $0.05 \mathrm{M}$ aniline. All chemicals were analytical grade. The electrodeposition was carried out at room temperature.

\section{Preparation of supercapacitors based on SWCNT/PANI hybrid films}

The freestanding hybrid films were cut into the desired shape and mass as supercapacitor electrodes. To calculate and compare specific capacitance conveniently, each SWCNT/PANI film supercapacitor electrode for $\mathrm{CV}$ and charge/discharge measurements has same mass $(10 \mu \mathrm{g})$. The electrolyte was $1 \mathrm{M}$ nonaqueous $\mathrm{LiClO}_{4}$ in a mixture of ethylene carbonate (EC), diethyl carbonate (DEC), and dimethylene carbonate (DMC) in a volume ratio of $\mathrm{EC} / \mathrm{DEC} / \mathrm{DMC}=1: 1: 1$. The separator (Celgard 2325) and electrolyte were sandwiched by the SWCNT/PANI hybrid films on the PET substrates. 

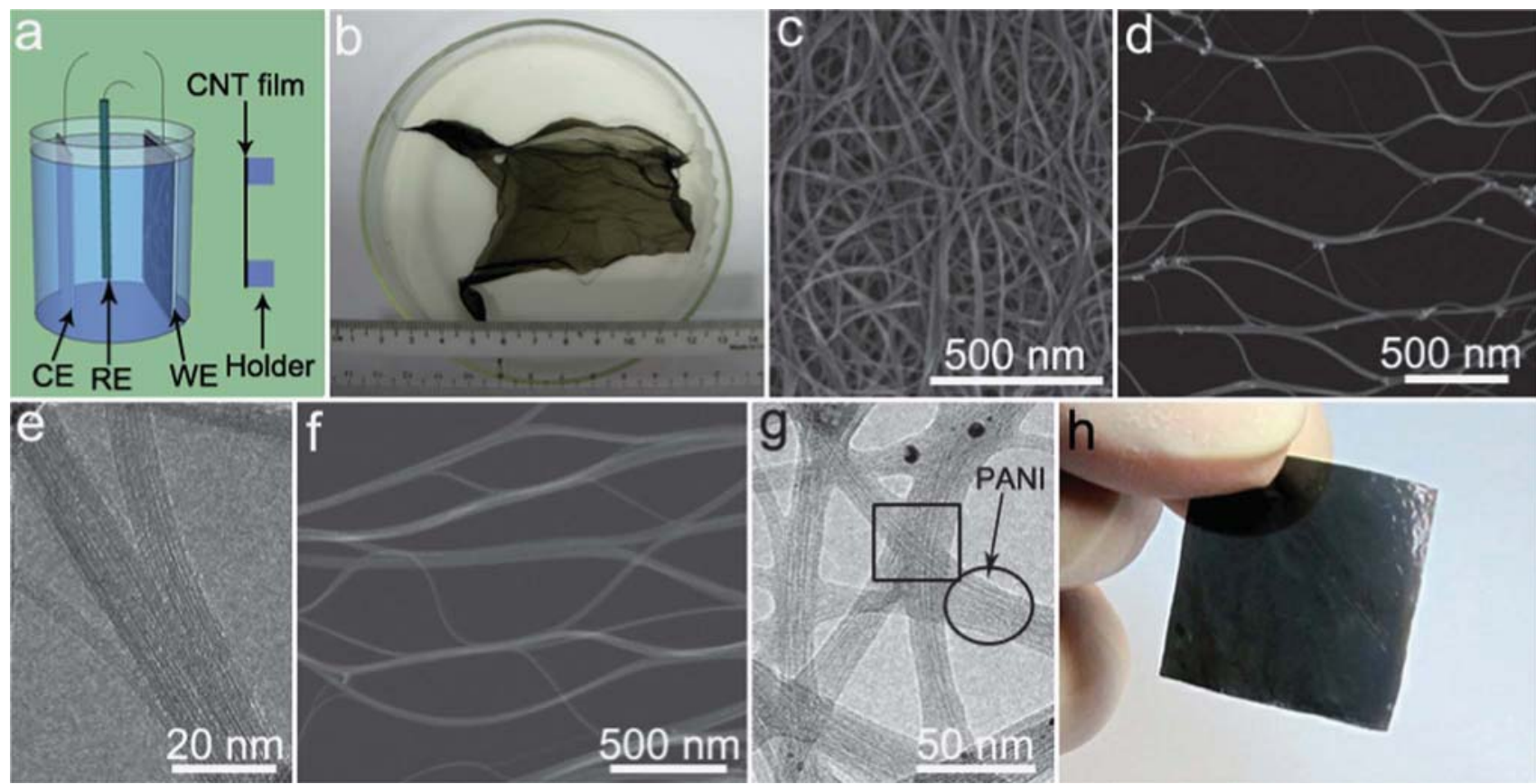

Fig. 1 (a) Sketch of PANI electrodeposition using the directly grown SWCNT film as template. (CE: counter electrode, RE: reference electrode, WE: working electrodes). (b) Optical image of a directly grown SWCNT film with about $200 \mathrm{~nm}$ thickness. (c) SEM image of a directly grown SWCNT film. (d) SEM image of a single layer of SWCNT bundles peeled off from a thick film. (e) TEM image of a SWCNT junction in the directly grown film. (f) SEM image of a single layer of SWCNT/PANI bundles peeled off from a thick SWCNT/PANI film. (g) TEM image of SWCNT/PANI in the hybrid film. (h) Optical image of SWCNT/PANI hybrid film (size: $2 \mathrm{~cm} \times 2 \mathrm{~cm}$, thickness: $\sim 240 \mathrm{~nm}$, deposition time: $30 \mathrm{~s}$ ).

\section{Characterization}

Cyclic voltammetry (CV) of the SWCNT/PANI hybrid film supercapacitors was measured by CHI 660C (CHI Instruments). The galvanostatic charge-discharge of the SWCNT film supercapacitors at a high operation voltage range $(0$ to $2.0 \mathrm{~V})$ was carried out on a supercapacitor test system (BT2000 Arbin). The morphology and microstructure of the SWCNT films or SWCNT/PANI hybrid films were characterized by scanning electron microscopy (SEM, Hitachi S-5200) and transmission electron microscopy (TEM, JEOL JEM-2010). The thickness of the SWCNT films and the SWCNT/PANI hybrid films was measured by atomic force microscopy (AFM, Dimension ${ }^{\circledR}$ Icon $($ ) with the NanoScope V Controller. The Raman spectra were recorded with a spectrophotometer (WITec alpha $300 \mathrm{R}$ ) with operating wavelengths of 633 and $532 \mathrm{~nm}$. FTIR spectra were recorded on a FT-IR system (Perkin Elmer).

\section{Results and discussion}

In situ electrochemical polymerization is an effective process to deposit PANI onto templates. It can sensitively adjust the morphology and microstructure of the PANI according to the template. In situ electrochemical polymerization of PANI was generally performed in a traditional three-electrode cell, as depicted in Fig. 1a. Here, we used free-standing directly grown SWCNT films as a template to prepare SWCNT/PANI hybrid films because of their high conductivity and unique structure. Free-standing directly grown SWCNT films possess homogeneity in about $50 \mathrm{~cm}^{2}$ (Fig. 1b), which provides an opportunity to tailor such SWCNT films into any desired shape as a template to electrodeposit PANI in accordance with the device requirement. Besides, the directly grown SWCNT film is highly conductive with a very low sheet resistance in the range of 5-50 Ohm per square for the film thickness from 500 to $100 \mathrm{~nm}$. This indicates that directly grown SWCNT films would be a good candidate as a template to deposit PANI. However, since the free-standing directly grown SWCNT films are thin (less than several hundred nanometers) and easily aggregated in electrolyte, they cannot be used directly as templates to deposit PANI. To overcome this problem, we spread out and fixed the film onto a holder with a hole (Fig. 1a), such that two sides of the film were in direct contact with the electrolyte. Although the directly grown SWCNT films are thin, these free-standing SWCNT films exhibits a high tensile strength of $360 \mathrm{MPa}$, which is 30 times higher than that of a typical bulky paper. ${ }^{44,45}$ Good strength and toughness ensure that the SWCNT films can be easily handled and can maintain its structure and integrity during the electrodeposition process, and act like a skeleton to support the deposited PANI layers. The SEM image of a SWCNT film (Fig. 1c) shows a nanoporous architecture without visible impurities, which would be of great significance for its use as a template to deposit PANI. The thinness and porous structure of the SWCNT films enable aniline molecules to infiltrate into the porous films easily and deposit onto the walls of SWCNT bundles more efficiently.

Fig. 1d displays a typical SEM image of single layer SWCNT bundles peeling off from a thick film. It clearly illustrates that the SWCNT bundles in the film are firmly connected with each other and form a continuous $2 \mathrm{D}$ reticulate structure in the plane parallel to the surface of the film. This unique architecture plays a key role in the higher strength and conductivity of the directly 
grown SWCNT films in comparison with other post-deposited CNT films, such as Bucky papers, ${ }^{44}$ which have short and randomly oriented CNTs. A TEM image of a SWCNT junction in a directly grown film (Fig. 1e) shows that the connection between SWCNTs in a single layer of SWCNT bundle network is continuous, interconnected, and not overlapping. This unique reticulate architecture has an advantage in transporting electrons over a large area. The electrodeposited PANI layer is coated on the surface of the SWCNT bundles with continuous reticulate architecture, maintaining the continuous reticulate architecture and thereby ensuring that the electrons are readily transported throughout the network, via the 'skeleton/skin' configuration as shown in Fig. If and 2a. The PANI layer, 'skin', on the surface of SWCNT bundles is also continuous, as shown in Fig. 1f and 2a. It replicates the reticulate structure of SWCNT bundles, which is helpful for electron transport. The junctions in the reticulate structure remain continuous, as marked by circles in Fig. 1g. In the normal direction of film, the continuous reticulate SWCNT bundles overlap to form the film. Since the surface of SWCNT bundles in the reticulate architecture is almost free of impurities (Fig. 1e), neighboring continuous reticulate SWCNT bundles are well-contacted by SWCNT-SWCNT surface contact due to van der Waals binding energy. After depositing the PANI layer, the PANI can also be well coated onto the surface of the junction, as marked by rectangle in Fig. 1g. Therefore, in the SWCNT/PANI hybrid film, the directly grown SWCNT film, i.e. the 'skeleton', could remain its original structure and the PANI layer, i.e., the 'skin', was uniform and continuously coated onto the surface of the SWCNT bundles in both parallel and normal direction of the film plane, forming a continuous 'skeleton/skin' structure. This structure is different from the conventional electrodes based on SWCNT/PANI powder and the SWCNT/PANI films based on post-deposited SWCNT films. For the SWCNT/PANI powder electrodes, first the PANI powder completely coated the individual SWCNT bundles, then the SWCNT/PANI powder was pressed into tablets to form electrodes. In the tablets, the connections between SWCNT bundles coated with a PANI layer is overlapped by PANI-PANI contacts and not continuous, as depicted in Fig. 2b. Hence, in the case of SWCNT/ PANI powder electrodes, electrons have to be transported through the overlapped PANI-PANI contact between SWCNT/ PANI bundles (depicted in Fig. 2d). For the SWCNT/PANI films based on post-deposited SWCNT films, the post-deposited SWCNT films have a short and random-oriented structure, which is different from directly grown SWCNT films. ${ }^{46-49}$ Besides, the surface of the SWCNT bundles in the post-deposited SWCNT film was generally coated by some molecular species used for making SWCNT suspensions. The molecular species would block the direct contact between SWCNT bundles and PANI layer and lead to relatively high contact resistance. In contrast, for our SWCNT/PANI hybrid films, electrons could be continuously transported through reticulate "skeleton/skin" architecture, i.e., the continuous SWCNT/PANI network, in which interbundle junctions and continuous connection ensure low resistance. Therefore, the conductivity of SWCNT/PANI films with a continuous "skeleton/skin" structure is much higher than that of SWCNT/PANI electrodes with overlapped PANIPANI contact.
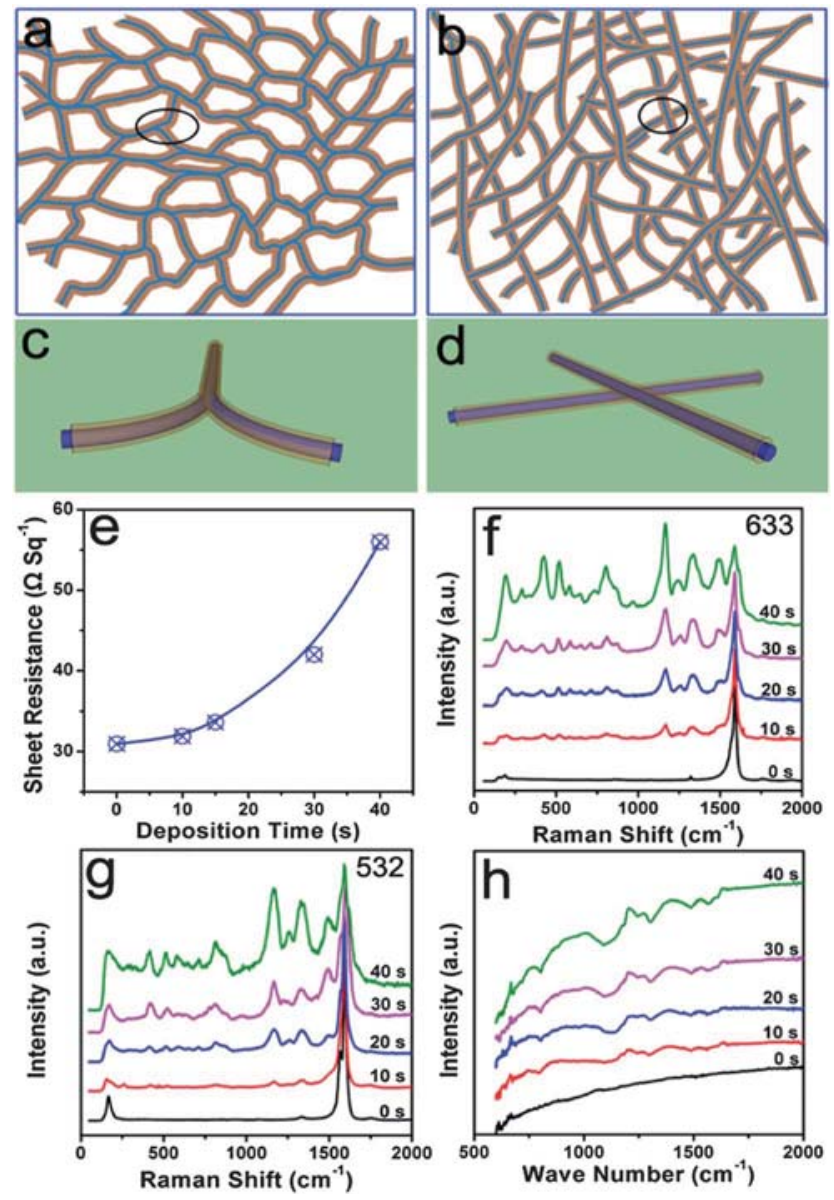

Fig. 2 The sketch diagrams of a single layer film based on (a) continuous SWCNT/PANI reticulate structure and (b) randomly overlapped SWCNT/PANI bundles. "Blue" and "brown" parts represent SWCNT bundle skeleton and PANI skin, respectively. The sketch diagrams of continuous interbundle SWCNT/PANI junctions (c) and overlapped SWCNT/PANI junctions (d). (e) The sheet resistance of the SWCNT/ PANI hybrid film under different PANI deposition time. (f) Raman spectra of the SWCNT/PANI hybrid film under different PANI deposition time at $\lambda=633$ (f) and $532 \mathrm{~nm}(\mathrm{~g})$. (h) FTIR spectra of the SWCNT/ PANI hybrid film under different PANI deposition time.

While the continuous SWCNT network in the SWCNT/PANI hybrid films is useful for transporting electrons and reducing the resistance, the deposition of PANI still affects the sheet resistance of the SWCNT/PANI hybrid films. The sheet resistance of the SWCNT/PANI hybrid films is increased with increasing PANI deposition time, as shown in Fig. 2e. Although the sheet resistance of the SWCNT/PANI hybrid film with 40 s PANI deposition time is about twice as high as that of the pure directly grown SWCNT film, its conductivity reaches about $1138 \mathrm{~S} \mathrm{~cm}^{-1}$, which is approximately 30 times higher than that reported for SWCNT/PANI hybrid films based on post-deposition SWCNT films $\left(20-35 \mathrm{~S} \mathrm{~cm}^{-1}\right),{ }^{30}$ and even higher than some pure SWCNT films prepared by post-deposited methods $\left(733 \mathrm{~S} \mathrm{~cm}^{-1}\right){ }^{44}$ The high conductivity of SWCNT/PANI hybrid films is attributed to the unique continuous "skeleton/skin" architecture, as discussed above. Since the thickness of SWCNT/PANI hybrid films is mainly dependent on the original SWCNT film template (about 
several hundred nanometers), which could be controlled by growth time, the thickness of SWCNT/PANI hybrid film is controllable and a thin free-standing SWCNT/PANI hybrid film can be achieved. However, for the SWCNT/PANI powder electrodes, because they were generally obtained by pressing the composites into tablets or mixing SWCNT/PANI composite with conductive binders and coating it onto collector electrodes, it is hard to obtain thin free-standing SWCNT/PANI hybrid electrodes. In addition, for the SWCNT/PANI film electrodes based on post-deposited SWCNT films, it is also difficult to obtain thin free-standing SWCNT/PANI film electrodes, due to the difficulty in obtaining thin free-standing post-deposited SWCNT films. Fig. $1 \mathrm{~h}$ is an optical image of a SWCNT/PANI hybrid film of about $240 \mathrm{~nm}$ in thickness. Furthermore, the films can be bent, rolled up and even twisted without cracking. Good mechanical properties, thinness and a porous structure make them suitable for application in flexible energy storage devices.

During the electrodeposition of PANI onto the directly grown SWCNT films, the PANI is continuously coated onto the surface of SWCNT bundles in the film. Thus, the content of PANI on the surface of SWCNT bundles would be increased with an increase in the PANI deposition time. Raman spectroscopy was used to characterize the change in the PANI content in the hybrid films, as shown in Fig. $2 \mathrm{f}$ and $\mathrm{g}$. To compare the relative change of peak intensities in Fig. 2f and g, the peaks at about $1590 \mathrm{~cm}^{-1}$ corresponding to $G$ band of SWCNTs were normalized to 1 . In Fig. 2f, the peaks ascribed to PANI were observed at about 425/ $518 \mathrm{~cm}^{-1}$ (C-C out-of-plane deformation), 804/867 $\mathrm{cm}^{-1}$ (outof-plane $\mathrm{C}-\mathrm{H}$ motion), $1165 \mathrm{~cm}^{-1}\left(\mathrm{C}-\mathrm{H}\right.$ bending), $1239 \mathrm{~cm}^{-1}$ $\left(\nu \mathrm{C}-\mathrm{N}\right.$ benzene diamine units) $1338 \mathrm{~cm}^{-1}\left(\nu \mathrm{C}-\mathrm{N}^{+}\right)$and 1492 $\mathrm{cm}^{-1}(\nu \mathrm{C}=\mathrm{N})$, indicating the formation of PANI onto the SWCNT films. ${ }^{25}$ Besides, it is clear that in Fig. 2f the relative intensities of peaks ascribed to PANI, such as $1165 \mathrm{~cm}^{-1}$, increased with the increase in PANI deposition time. This indicates that more and more PANI was electrodeposited onto the SWCNT film with the increase in PANI deposition time. Raman spectra at operating wavelength $\lambda=532 \mathrm{~nm}$ is similar to that at $\lambda=633 \mathrm{~nm}$ (Fig. $2 \mathrm{~g}$ ). The peaks ascribed to PANI were observed and their relative intensities also increased with the increase in PANI deposition time. The conclusions derived from Raman spectroscopy were corroborated by FTIR spectroscopy (Fig. 2h). The significant increase in the intensity of adsorption bands corresponding to PANI $\left(1095 \mathrm{~cm}^{-1}\right.$ due to $\nu \mathrm{C}-\mathrm{N}^{+}=\mathrm{C}$, $1244 \mathrm{~cm}^{-1}$ due to $\nu \mathrm{C}-\mathrm{N}^{+}$, and $1302 \mathrm{~cm}^{-1}$ due to $\nu \mathrm{C}-\mathrm{N}, 1486$ $\mathrm{cm}^{-1}$ due to $\nu \mathrm{C}-\mathrm{C}$ in benzenoid rings, $1570 \mathrm{~cm}^{-1}$ due to $\nu \mathrm{C}-\mathrm{C}$ in quinoid rings) indicated the formation of PANI on the SWCNT film and that more and more PANI was electrodeposited onto the SWCNT film with the increase in PANI deposition time. ${ }^{25}$ During the electrodeposition of PANI, the diameter of the SWCNT bundles coated by PANI increased. The pores were filled with the increase in PANI deposition time, as shown in Fig. 3. From Fig. 3, it can be seen that, when the deposition time is less than $30 \mathrm{~s}$ (Fig. 3d), the porous structure of the SWCNT/ PANI hybrid film could be maintained. This is significant for the diffusion of electrolyte into the SWCNT/PANI hybrid film. However, when the deposition time was increased to $40 \mathrm{~s}$, some pores of the film were filled (Fig. 3e). Finally, at $50 \mathrm{~s}$, all the pores were almost filled and the porous structure disappeared, as shown in Fig. $3 f$.
Fig. 4 shows the TEM images of SWCNT bundles and SWCNT bundles coated with PANI layers formed with different deposition times. The wall of the pure SWCNT bundle is clean, as shown in Fig. 4a. During electrodeposition, the PANI could effectively coat the surface of the SWCNT bundles, as shown in Fig. $4 b-d$, which is consistent with the SEM results. With an increase in the PANI deposition time, the thickness of PANI layer on the surface of SWCNT bundle was increased and uniform (Fig. 4b-d).

In situ electrochemical polymerization realized the effective deposition of PANI onto the surface of SWCNT bundles in the film and controlled the morphology and microstructure of the SWCNT/PANI hybrid film, forming a continuous and porous "skeleton/skin" architecture and offering a good compromise between pore density and amount of PANI. Continuous "skeleton/skin" architecture ensures that the SWCNT/PANI hybrid films possess high conductivity. Moreover, thin SWCNT/PANI hybrid films remain free-standing and can be bent, rolled up and even twisted without cracking. As a promising carbon material for flexible supercapacitors, ${ }^{50-56}$ the as-prepared thin SWCNT/ PANI hybrid films, which combine high conductivity from SWCNT films and the pseudocapacitance from PANI, are suitable for the electrodes of flexible supercapacitors, in which neither an insulating binder nor a low capacitance conducting additive is required.

As a proof-of-concept, we constructed and measured the performance of two-electrode symmetrical flexible supercapacitors based on the thin free-standing SWCNT/PANI hybrid films. Fig. 5a is the schematic diagram of a flexible SWCNT/ PANI hybrid film supercapacitor. The separator and electrolyte were sandwiched by the SWCNT/PANI hybrid films on the PET substrates. The SWCNT/PANI hybrid films were directly used as
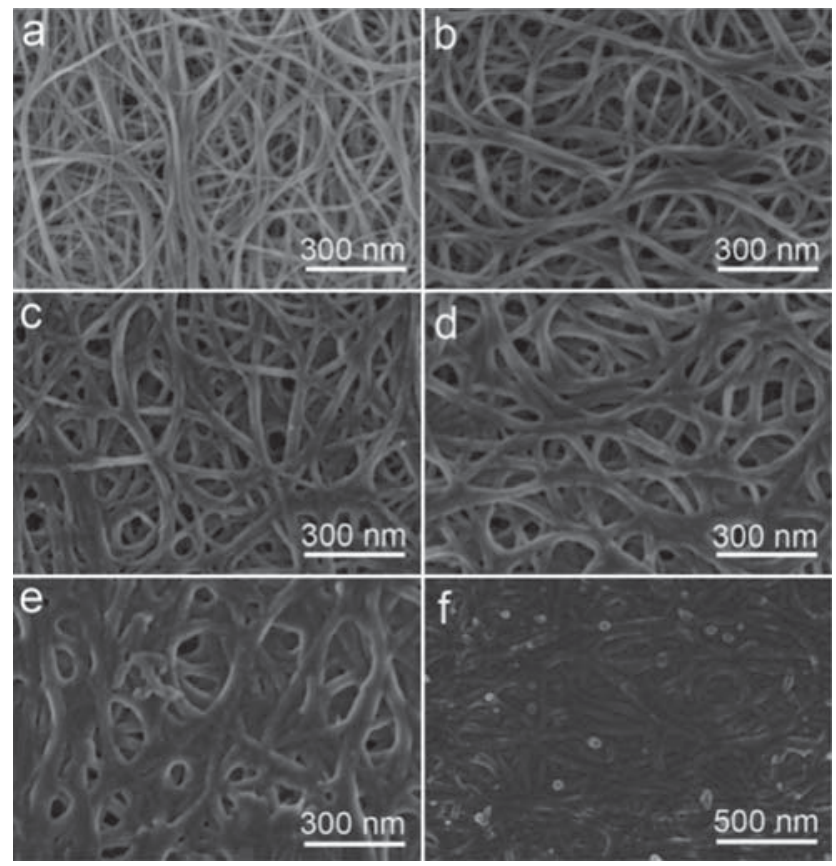

Fig. 3 The SEM images of the SWCNT/PANI hybrid films formed with different PANI deposition times: (a) pure SWCNT film, (b) $10 \mathrm{~s}$, (c) $20 \mathrm{~s}$, (d) $30 \mathrm{~s}$, (e) $40 \mathrm{~s}$, and (f) $50 \mathrm{~s}$. 

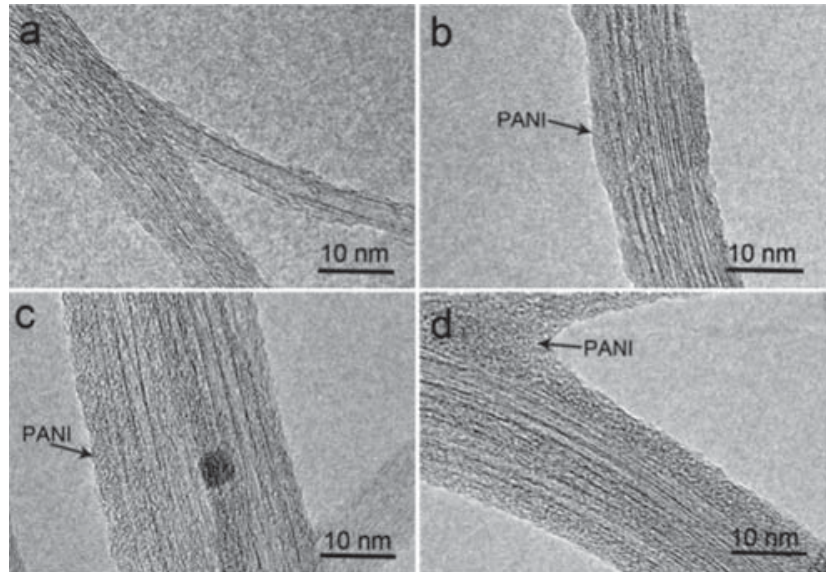

Fig. 4 The TEM images of the SWCNT/PANI hybrid films under different PANI deposition time: (a) pure SWCNT bundle, (b) $10 \mathrm{~s}$, (c) $20 \mathrm{~s}$, and (d) $30 \mathrm{~s}$.

both electrodes and charge collectors to prepare supercapacitors, simplifying the architecture and reducing the weight. The inset in Fig. 5a is an optical image of a flexible SWCNT/PANI hybrid film supercapacitor.

The electrochemical characterization of supercapacitors based on the pure SWCNT film and SWCNT/PANI hybrid films were investigated by CVs (Fig. 5b). The CV of supercapacitor based on the pure SWCNT film shows a typical rectangular shape, suggesting that it is an electrochemical double-layer capacitor, which is similar to the results obtained from other pure SWCNTbased supercapacitors. ${ }^{15}$ The specific capacitance of pure SWCNT film-based supercapacitors is only about $23.5 \mathrm{~F} \mathrm{~g}^{-1}$. However, the CV curve area of the SWCNT/PANI hybrid film is much larger than that of the pure SWCNT film, which indicates that the specific capacitance of the SWCNT/PANI hybrid film is much higher than that of the pure SWCNT film, owing to its additional pseudocapacitance. The CV curve area of SWCNT/ PANI hybrid film with $30 \mathrm{~s}$ deposition time is higher than that of other SWCNT/PANI hybrid films, demonstrating that the corresponding specific capacitance of the SWCNT/PANI hybrid film with $30 \mathrm{~s}$ deposition time is optimal. Fig. 5c shows the CV curves of a representative SWCNT/PANI optimized hybrid film (30 s) supercapacitor at different scan rates. It can be seen that CVs of the supercapacitor keep their shape within a selected range of potential and even at a high scan rate of $500 \mathrm{mV} \mathrm{s}^{-1}$, indicating that the SWCNT/PANI hybrid films have very rapid current response on voltage reversal.

For supercapacitors based on organic electrolyte, $2 \mathrm{~V}$ is generally used as the applied voltage to guarantee both wide electrochemical window and stable electrochemical performance. So, the charge-discharge curves of the SWCNT/PANI hybrid film supercapacitor cycled between 0 and $2.0 \mathrm{~V}$ at a constant current density of $10 \mathrm{~A} \mathrm{~g}^{-1}$ are measured, as shown in Fig. $5 \mathrm{~d}$. The shape of charge-discharge curves of SWCNT/PANI hybrid film supercapacitors is similar to the reported results. ${ }^{31}$ The discharge curves were not an ideal straight line, indicating the process of a faradic reaction. ${ }^{17}$

The specific capacitances $C_{\text {spe }}$ of supercapacitor devices were calculated from constant current charge-discharge curves using the following equation ${ }^{15}$
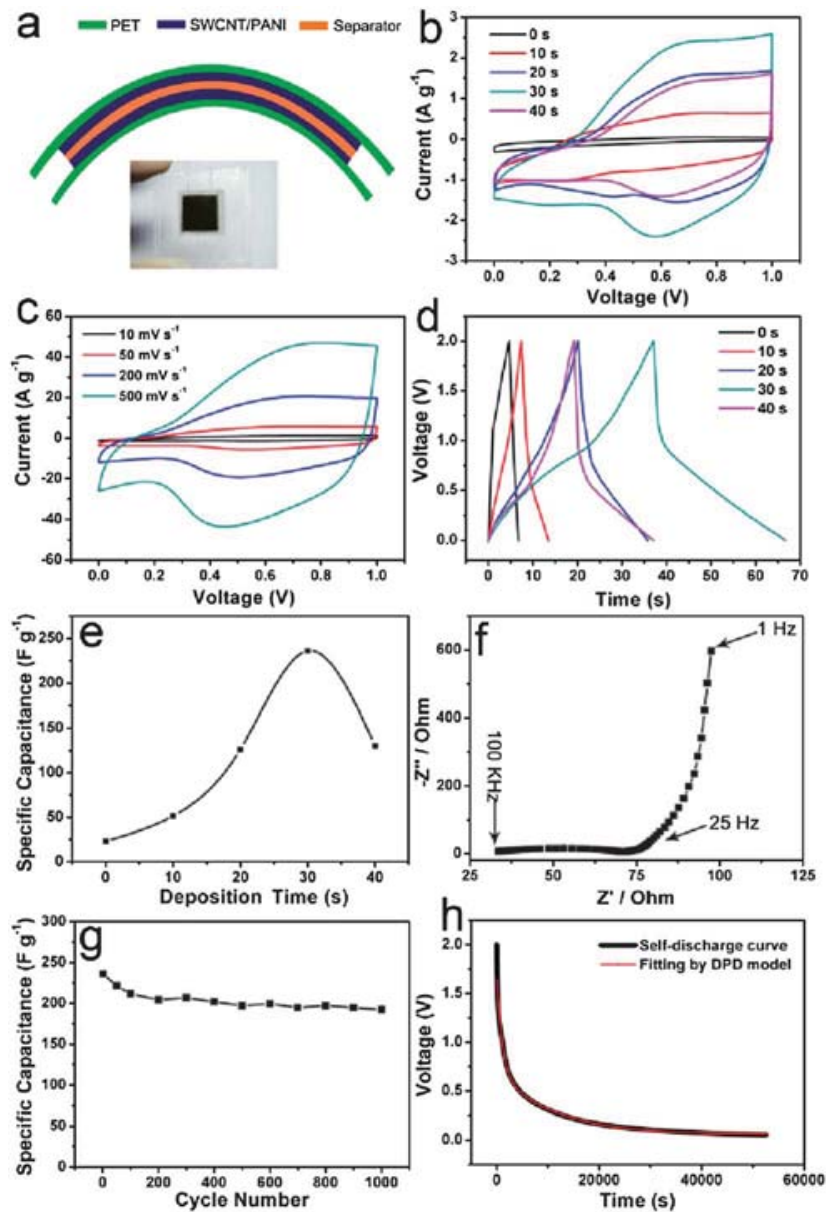

Fig. 5 (a) Schematic diagram and optical image of a flexible SWCNT/ PANI hybrid film supercapacitor. (b) CV curves of supercapacitors based on SWCNT/PANI hybrid film under different PANI deposition time, scan rate: $20 \mathrm{mV} \mathrm{s}^{-1}$ (c) $\mathrm{CVs}$ of SWCNT/PANI hybrid film supercapacitors at different scan rates. (d) Charge-discharge curves and (e) specific capacitance of supercapacitors based on SWCNT/PANI hybrid film under different PANI deposition time. (f) Nyquist impedance plots of the SWCNT/PANI hybrid film supercapacitor with frequency ranging from $100 \mathrm{kHz}$ to $1 \mathrm{~Hz}$. (g) Variation of capacitance stability with cycle number of a SWCNT/PANI hybrid film supercapacitor. (h) The selfdischarge and fitting curves of the SWCNT/PANI hybrid film supercapacitor.

$$
C_{\text {spe }}=\frac{I}{(\mathrm{~d} V / \mathrm{d} t) m}
$$

where $I$ is the discharge current, $\mathrm{d} V / \mathrm{d} t$ represents the slope of the discharge curve and $m$ is the total mass of the SWCNT/PANI hybrid films on both electrodes.

Fig. 5e shows the calculated specific capacitances of supercapacitors using SWCNT/PANI hybrid films under different PANI deposition times as electrodes. The specific capacitance of SWCNT/PANI hybrid film with 30 s PANI deposition time is about $236 \mathrm{~F} \mathrm{~g}^{-1}$, which is much larger than the pure SWCNT film $\left(23.5 \mathrm{~F} \mathrm{~g}^{-1}\right)$, indicating that the specific capacitance of SWCNT film was remarkably improved due to the pseudocapacitance contributed by PANI. Fig. 5e demonstrates that, when the PANI deposition time is shorter than $30 \mathrm{~s}$, the specific capacitance of SWCNT/PANI hybrid film electrode is increased 
with the deposition time. However, when the PANI deposition time was longer than $30 \mathrm{~s}$, the specific capacitance of SWCNT/ PANI hybrid film electrode is decreased with increases in the deposition time. As discussed above, with an increase in deposition time, the thickness of PANI layer is increased. So, specific capacitance of the SWCNT/PANI hybrid film is increased with an increase in PANI deposition time during the initial $30 \mathrm{~s}$ because increased PANI would provide more pseudocapacitance. However, when the deposition time was longer than $30 \mathrm{~s}$, some pores of the SWCNT/PANI hybrid films were filled (Fig. 3). As a result, the electrolyte cannot diffuse into the interior of the SWCNT/PANI hybrid film, leading to a low specific capacitance. This result is consistent with the $\mathrm{CV}$ analysis.

The energy density ( $W=C_{\text {spe }} V^{2} / 2$, where $V$ is the voltage applied) of SWCNT/PANI hybrid film electrodes for the asassembled supercapacitor is $131 \mathrm{~W} \mathrm{~h} \mathrm{~kg}^{-1}$ and their maximum power density $\left(P_{\max }=V^{2} / 4 R m\right.$, where $R$ is the internal resistance, $m$ is the total mass of two electrodes) is $62.5 \mathrm{~kW} \mathrm{~kg}^{-1}$, which is much higher than the case of supercapacitors based on CNT/PANI powder $\left(<2.5 \mathrm{~kW} \mathrm{~kg}^{-1}\right)^{43,57}$ and films $\left(<2.2 \mathrm{~kW} \mathrm{~kg}^{-1}\right)$. $^{31,41}$

Further electrochemical investigation was carried out for in depth understanding of the SWCNT/PANI hybrid film supercapacitor device performance. The impedance spectrum of the SWCNT/PANI hybrid film supercapacitor is shown as a Nyquist plot in Fig. 5f. In the high frequency domain, a small semi-circle is observed (bulk RC response), which can be attributed to contact impedance generated between the SWNTs and deposited PANI as well as electrolyte resistance within the pores of the composite material. ${ }^{58}$ In the low frequency portion of the spectrum, the plot tends towards a vertical line where the imaginary part of impedance rapidly increases. This is characteristic of capacitive behavior, and representative of the ion diffusion in the electrode structure. ${ }^{52}$ The knee frequency is usually used to evaluate the frequency dependence of a capacitor and considered to be the critical frequency where supercapacitors begin to exhibit capacitive behavior. In Fig. 5f, it can be seen that the knee frequency is $25 \mathrm{~Hz}$, indicating that most of its stored energy is accessible at frequencies below $25 \mathrm{~Hz}$ for the SWCNT/PANI hybrid film supercapacitors.

In order to study the stability of SWCNT/PANI film supercapacitors, the as-prepared supercapacitor was examined by galvanostatic charge-discharge measurements for 1000 cycles under the same conditions, as shown in Fig. 5g. With an increase in the cycle number, the specific capacitance decreased, losing about $15 \%$ of the maximum capacity after 100 cycles, and then remained almost unchanged. Swelling and shrinkage of conducting polymers when used as electrodes is well known and may lead to degradation of the electrode during cycling. This could be overcome, to some extent, by the "skeleton/skin" structure of SWCNT/PANI hybrid films. The self-discharge (SDC) behaviour of supercapacitors is an important factor for energy retention. ${ }^{59,60}$ The SDC of the SWCNT/PANI hybrid film supercapacitor was measured and is shown in Fig. 5h. The supercapacitor undergoes a rapid SDC at the beginning, and then the SDC rate gradually becomes smaller and more stable. Wei et al. suggested that a divided potential driving (DPD) model can be used to fit the SDC behaviour of SWCNT-based supercapacitors. ${ }^{60}$ The fitting to our measurements is satisfactory, as shown in Fig. 5h, indicating that the divided potential driving force plays an important role in the SDC process for as-prepared SWCNT/PANI hybrid film supercapacitors. ${ }^{60}$

\section{Conclusion}

Free-standing, ultrathin and flexible SWCNT/PANI hybrid films with unique continuous "skeleton/skin" structure were prepared by a simple in situ electrochemical polymerization method. In situ electrochemical polymerization realizes an effective deposition of PANI onto the surface of SWCNT bundles in the films and controls the morphology and microstructure of the SWCNT/PANI hybrid films, forming continuous and porous "skeleton/skin" architecture and offering a good compromise between pore density and the amount of PANI. Compared to the SWCNT/PANI composite films based on post-deposition SWCNT films, the thin hybrid films have high conductivity due to their unique continuous 'skeleton/skin' reticulate architecture. Furthermore, they can be bent, rolled up and even twisted without cracking. The high electrical conductivity and flexibility, in combination with the continuous porous structure, suggest that the free-standing SWCNT/ PANI hybrid films have significant potential as promising electrode materials for thin, lightweight and flexible energy storage devices with high performance. The electrochemical properties of the SWCNT/PANI hybrid film electrodes were modulated by controlling pore density, which was a key factor affecting the performance of as-assembled supercapacitors. Flexible supercapacitors were fabricated using thin free-standing SWCNT/PANI hybrid films as both electrodes and charge collectors without metallic current collectors, in which neither an insulating binder nor a low capacitance conducting additive is required. High energy and power densities ( $131 \mathrm{~W} \mathrm{~h} \mathrm{~kg}^{-1}$ and $62.5 \mathrm{~kW} \mathrm{~kg}^{-1}$, respectively) were achieved for the as-prepared flexible supercapacitors based on the optimized SWCNT/PANI hybrid films.

\section{Acknowledgements}

This work is supported by the National Basic Research Program of China (Grant no. 2012CB932302), the National Natural Science Foundation of China (51172271, and 90921012), and the Beijing Municipal Education Commission (Grant no. YB20108000101).

\section{Notes and references}

1 P. Simon and Y. Gogotsi, Nat. Mater., 2008, 7, 845-854.

2 Y. Zhang, H. Feng, X. B. Wu, L. Z. Wang, A. Q. Zhang, T. C. Xia, H. C. Dong, X. F. Li and L. S. Zhang, Int. J. Hydrogen Energy, 2009, 34, 4889-4899.

3 L. L. Zhang and X. S. Zhao, Chem. Soc. Rev., 2009, 38, 2520-2531. 4 C. Liu, F. Li, L. P. Ma and H. M. Cheng, Adv. Mater., 2010, 22, E28E62.

5 S. X. Xiong, J. Wei, P. T. Jia, L. P. Yang, J. Ma and X. H. Lu, ACS Appl. Mater. Interfaces, 2011, 3, 782-788.

6 Z. Q. Niu, J. Chen, H. H. Hng, J. Ma and X. D. Chen, Adv. Mater., 2012, 24, 4144 4150.

7 Y. Y. Liang, M. G. Schwab, L. J. Zhi, E. Mugnaioli, U. Kolb, X. L. Feng and K. Mullen, J. Am. Chem. Soc., 2010, 132, 1503015037.

8 K. H. An, W. S. Kim, Y. S. Park, Y. C. Choi, S. M. Lee, D. C. Chung, D. J. Bae, S. C. Lim and Y. H. Lee, Adv. Mater., 2001, 13, 497-500.

9 A. Izadi-Najafabadi, S. Yasuda, K. Kobashi, T. Yamada, D. N. Futaba, H. Hatori, M. Yumura, S. Iijima and K. Hat, $A d v$. Mater., 2010, 22, E235-E241. 
10 C. M. Niu, E. K. Sichel, R. Hoch, D. Moy and H. Tennent, Appl. Phys. Lett., 1997, 70, 1480-1482.

11 M. Kaempgen, C. K. Chan, J. Ma, Y. Cui and G. Gruner, Nano Lett., 2009, 9, 1872-1876.

12 L. B. Hu, J. W. Choi, Y. Yang, S. Jeong, F. La Mantia, L. F. Cui and Y. Cui, Proc. Natl. Acad. Sci. U. S. A., 2009, 106, 21490-21494.

13 V. L. Pushparaj, M. M. Shaijumon, A. Kumar, S. Murugesan, L. Ci, R. Vajtai, R. J. Linhardt, O. Nalamasu and P. M. Ajayan, Proc. Natl. Acad. Sci. U. S. A., 2007, 104, 13574-13577.

14 A. Izadi-Najafabadi, T. Yamada, D. N. Futaba, M. Yudasaka, H. Takagi, H. Hatori, S. Iijima and K. Hata, ACS Nano, 2011, 5, 811-819.

15 Z. Q. Niu, W. Y. Zhou, J. Chen, G. X. Feng, H. Li, W. J. Ma, J. Z. Li, H. B. Dong, Y. Ren, D. Zhao and S. S. Xie, Energy Environ. Sci, 2011, 4, 1440-1446.

16 G. Wee, T. Salim, Y. M. Lam, S. G. Mhaisalkar and M. Srinivasan, Energy Environ. Sci., 2011, 4, 413-416.

17 J. Yan, T. Wei, Z. J. Fan, W. Z. Qian, M. L. Zhang, X. D. Shen and F. Wei, J. Power Sources, 2010, 195, 3041-3045.

18 H. L. Zhang, D. C. Wei, Y. Q. Liu, B. Wu, L. P. Huang, H. X. Xi J. Y. Chen, G. Yu, H. Kajiura and Y. M. Li, Small, 2009, 5, 23922396.

19 P. C. Chen, G. Z. Shen, Y. Shi, H. T. Chen and C. W. Zhou, ACS Nano, 2010, 4, 4403-4411.

20 J. H. Kim, K. H. Lee, L. J. Overzet and G. S. Lee, Nano Lett., 2011, 11, 2611-2617.

21 X. Li, J. P. Rong and B. Q. Wei, ACS Nano, 2010, 4, 6039-6049.

22 C. J. Yu, C. Masarapu, J. P. Rong, B. Q. Wei and H. Q. Jiang, $A d v$. Mater., 2009, 21, 4793-4797.

23 V. V. N. Obreja, Phys. E., 2008, 40, 2596-2605.

24 M. N. Hyder, S. W. Lee, F. C. Cebeci, D. J. Schmidt, Y. Shao-Horn and P. T. Hammond, ACS Nano, 2011, 5, 8552-8561.

25 R. V. Salvatierra, M. M. Oliveira and A. J. G. Zarbin, Chem. Mater., 2010, 22, 5222-5234

26 A. A. Mikhaylova, E. K. Tusseeva, N. A. Mayorova, A. Y. Rychagov, Y. M. Volfkovich, A. V. Krestinin and O. A. Khazova, Electrochim. Acta, 2011, 56, 3656-3665.

27 T. Wang, A. Kiebele, J. Ma, S. Mhaisalkar and G. Gruner, J. Electrochem. Soc., 2011, 158, A1-A5.

28 J. Ge, G. H. Cheng and L. W. Chen, Nanoscale, 2011, 3, 3084 3088.

29 J. E. Huang, X. H. Li, J. C. Xu and H. L. Li, Carbon, 2003, 41, 27312736 .

30 J. L. Liu, J. Sun and L. Gao, Nanoscale, 2011, 3, 3616-3619.

31 C. Z. Meng, C. H. Liu and S. S. Fan, Electrochem. Commun., 2009, 11, 186-189.

32 T. M. Wu, Y. W. Lin and C. S. Liao, Carbon, 2005, 43, 734-740.

33 J. A. Lee, M. K. Shin, S. H. Kim, S. J. Kim, G. M. Spinks, G. G. Wallace, R. Ovalle-Robles, M. D. Lima, M. E. Kozlov and R. H. Baughman, ACS Nano, 2012, 6, 327-334.

34 L. Y. Yuan, X. H. Lu, X. Xiao, T. Zhai, J. J. Dai, F. C. Zhang, B. Hu, X. Wang, L. Gong, J. Chen, C. G. Hu, Y. X. Tong, J. Zhou and Z. L. Wang, ACS Nano, 2012, 6, 656-661.
35 C. Peng, S. W. Zhang, D. Jewell and G. Z. Chen, Prog. Nat. Sci. 2008, 18, 777-788

36 Y. Z. Liao, C. Zhang, Y. Zhang, V. Strong, J. S. Tang, X. G. Li, K. Kalantar-zadeh, E. M. V. Hoek, K. L. Wang and R. B. Kaner, Nano Lett., 2011, 11, 954-959.

37 H. L. Wang, Q. L. Hao, X. J. Yang, L. D. Lu and X. Wang, Nanoscale, 2010, 2, 2164-2170.

38 X. B. Yan, Z. X. Tai, J. T. Chen and Q. J. Xue, Nanoscale, 2011, 3, 212-216.

39 H. S. Fan, H. Wang, N. Zhao, X. L. Zhang and J. Xu, J. Mater. Chem., 2012, 22, 2774-2780.

40 Q. Wu, Y. X. Xu, Z. Y. Yao, A. R. Liu and G. Q. Shi, ACS Nano, 2010, 4, 1963-1970.

41 C. Z. Meng, C. H. Liu, L. Z. Chen, C. H. Hu and S. S. Fan, Nano Lett., 2010, 10, 4025-4031.

42 Y. Zhou, Z. Y. Qin, L. Li, Y. Zhang, Y. L. Wei, L. F. Wang and M. F. Zhu, Electrochim. Acta, 2010, 55, 3904-3908.

43 M. G. Deng, B. C. Yang and Y. D. Hu, J. Mater. Sci., 2005, 40, 50215023.

44 D. H. Zhang, K. Ryu, X. L. Liu, E. Polikarpov, J. Ly, M. E. Tompson and C. W. Zhou, Nano Lett., 2006, 6, 1880-1886.

45 W. J. Ma, L. Song, R. Yang, T. H. Zhang, Y. C. Zhao, L. F. Sun, Y. Ren, D. F. Liu, L. F. Liu, J. Shen, Z. X. Zhang, Y. J. Xiang, W. Y. Zhou and S. S. Xie, Nano Lett., 2007, 7, 2307-2311.

46 W. Y. Zhou, W. J. Ma, Z. Q. Niu, L. Song and S. S. Xie, Chin. Sci. Bull., 2012, 57, 205-224.

47 Z. Q. Niu, W. J. Ma, H. B. Dong, J. Z. Li and W. Y. Zhou, Chin. Phys. B, 2011, 20, 028101.

48 J. Z. Li, W. J. Ma, L. Song, Z. G. Niu, L. Cai, Q. S. Zeng, X. X. Zhang, H. B. Dong, D. Zhao, W. Y. Zhou and S. S. Xie, Nano Lett., 2011, 11, 4636-4641.

49 J. Z. Li, Y. Gao, W. J. Ma, L. Q. Liu, Z. Zhang, Z. Q. Niu, Y. Ren, X. X. Zhang, Q. S. Zeng, H. B. Dong, D. Zhao, L. Cai, W. Y. Zhou and S. S. Xie, Nanoscale, 2011, 3, 3731-3736.

50 K. Zhang, L. L. Zhang, X. S. Zhao and J. S. Wu, Chem. Mater., 2010, 22, 1392-1401.

51 L. L. Zhang, R. Zhou and X. S. Zhao, J. Mater. Chem., 2010, 20, 5983-5992.

52 C. G. Liu, Z. N. Yu, D. Neff, A. Zhamu and B. Z. Jang, Nano Lett., 2010, 10, 4863-4868.

53 M. D. Stoller, S. J. Park, Y. W. Zhu, J. H. An and R. S. Ruoff, Nano Lett., 2008, 8, 3498-3502.

54 Y. Wang, Z. Q. Shi, Y. Huang, Y. F. Ma, C. Y. Wang, M. M. Chen and Y. S. Chen, J. Phys. Chem. C, 2009, 113, 13103-13107.

55 D. S. Yu and L. M. Dai, J. Phys. Chem. Lett., 2010, 1, 467-470.

56 Z. J. Fan, J. Yan, L. J. Zhi, Q. Zhang, T. Wei, J. Feng, M. L. Zhang, W. Z. Qian and F. Wei, Adv. Mater., 2010, 22, 3723-3728.

57 V. Gupta and N. Miura, Electrochim. Acta, 2006, 52, 1721-1726.

58 H. Kurig, A. Janes and E. Lust, J. Electrochem. Soc., 2010, 157, A $272-\mathrm{A} 279$.

59 Q. Zhang, J. P. Rong, D. S. Ma and B. Q. Wei, Energy Environ. Sci. 2011, 4, 2152-2159.

60 Q. Zhang, J. P. Rong and B. Q. Wei, RSC Adv., 2011, 1, 989-994. 